\title{
Enfant sujet, enfant objet? L'enfant comme enjeu des nouvelles politiques pénales au Québec, de la seconde moitié du XIX siècle au début du $X X$ siècle
}
Is the Child a Subject or an Object? The Child as the Focus of New Policies on Criminality in Quebec, from the Second Half of the 19th Century to the Beginning of the 20th Century El niño como sujeto, el niño como objeto ? El niño dentro de las nuevas políticas penales en Quebec, de la segunda mitad del siglo XIX a principios del siglo XX

\section{Sylvie MÉNARD et Véronique STRIMELLE}

\section{Numéro 44, automne 2000}

L'enfant au coeur des politiques sociales

URI : https://id.erudit.org/iderudit/005188ar

DOI : https://doi.org/10.7202/005188ar

\section{Aller au sommaire du numéro}

\section{Éditeur(s)}

Lien social et Politiques

ISSN

1204-3206 (imprimé)

1703-9665 (numérique)

\section{Découvrir la revue}

Citer cet article

MÉNARD, S. \& STRIMELLE, V. (2000). Enfant sujet, enfant objet? L'enfant comme enjeu des nouvelles politiques pénales au Québec, de la seconde moitié du XIX ${ }^{\mathrm{e}}$ siècle au début du XX ${ }^{\mathrm{e}}$ siècle. Lien social et Politiques, (44), 89-99. https://doi.org/10.7202/005188ar
Résumé de l'article

Dans la plupart des pays occidentaux, c'est au cours du XIXe siècle que sont élaborées les premières politiques protectrices de l'enfance délinquante et en danger. L'article décrit les différentes étapes qui ont conduit au Québec à l'adoption d'un régime spécifique destiné d'abord à l'enfance délinquante puis à toute l'enfance jugée « malheureuse ». Faire l'histoire des politiques destinées à l'enfance déviante conduit inéluctablement à examiner la notion même d'enfance et ses transformations. L'enfance va devenir à la fin du XIXe siècle un enjeu capital dans la stratégie mise au point par l'État pour prendre en charge des franges de population qui échappaient jusqu'alors à son emprise. 


\section{Enfant sujet, enfant objet ? L'enfant comme enjeu des nouvelles politiques pénales au Québec, de la seconde moitié du $\mathrm{XIX}^{\mathrm{e}}$ siècle au début du $\mathrm{XX}^{\mathrm{e}}$ siècle}

\section{Sylvie Ménard et Véronique Strimelle}

La mise en place d'institutions d'enfermement de plus en plus spécialisées est un phénomène qui a marqué la plupart des sociétés occidentales au cours du $\mathrm{XIX}^{\mathrm{e}}$ siècle $^{1}$. À cette époque, la reconnaissance de diverses formes de déviance suscite l'apparition de modes de traitement plus adaptés aux problèmes à traiter. Par la suite, la prise en charge se spécialisera aussi en fonction des populations concernées: les femmes seront

LIEN SOCIAL ET POLITIQUES - RIAC, 44 AUTOMNE 2000 séparées des hommes dans les institutions d'enfermement, et les déteruus plus jeunes ne seront plus placés dans les mêmes institutions que les adultes. Ainsi apparaissent, dans la seconde moitié du $\mathrm{XIX}^{\mathrm{e}}$ siècle, les premiers établissements spéciaux destinés aux enfants (Carlier, 1994; Petit, 1990 : 18-180).

Qu'il vive en milieu urbain ou rural, aisé ou pauvre, l'enfant était perçu: comme un adulte en réduction qui partageait le bon ou le mauvais sort de ses aînés. Hormis quelques écoles non obligatoires accueillant un nombre infime d'enfants pauvres, il n'existait pas de lieux propres à l'enfance (Dupont-Bouchat et al., 1996: 10; Gubin, 1984: 3).

La prise de conscience de la spécificité de l'enfance apparaît notamment dans le cadre des prisons. $\grave{A}$ partir des années 1830840 , quelques philanthropes et réformateurs pénitentiaires dénoncent les effets dévastateurs de la promiscuité entre enfants et adultes dans les lieux d'enfermement ${ }^{2}$. Cette prise de conscience aboutira en Europe et en Amérique du Nord 
LIEN SOCIAL ET POLITIQUES - RIAC, 44

Enfant sujet, enfant objet? L'enfant comme enjeu des nouvelles politiques pénales au Québec, de la seconde moitié du $X I X{ }^{c}$ siècle au début du XX $X^{e}$ siècle

à la création des premières institutions pour enfants délinquants.

Ce besoin de séparer les enfants des adultes révèle des préoccupations nouvelles pour la sauvegarde des enfants menacés par l'industrialisation et l'urbanisation croissantes. Mais les mesures de protection ne visent pas leur seul bien-être, ou la résolution de certains problèmes sociaux. Elles manifestent plutôt le désir d'intervenir auprès des familles pour mieux les contrôler et les intégrer aux exigences de la société industrielle. Dans cette perspective, l'apparition de la notion d'enfance et des mesures destinées à la protéger ou à la corriger est un exemple frappant du processus de récupération et de régulation de l'espace social par le pouvoir politique. Sous cet angle, la «découverte de l'enfance» ne vise pas tant à faire de l'enfant un sujet de droit qu'à en faire un objet de régulation (Fecteau et al., 1998: 76-77; Ménard, 1998a: 13; Strimelle, 1998: 116-117).

$\mathrm{Au}$ Québec, avant les années 1850 , peu de gens se préoccupent du sort des enfants malheureux, et c'est dans le cadre des prisons que vont se penser les premières ébauches d'une politique de l'enfance. On commence par séparer les enfants des adultes dans les établissements pénitentiaires grâce à la création, en 1857 , d'une prison de réforme à l'l̂le-aux-Noix ${ }^{3}$. Par la suite, on distingue les enfants délinquants des enfants abandonnés. La création des écoles de réforme et d'industrie, en 1869, vient concrétiser cette différenciation. C'est alors aussi que la loi établit un réseau d'institutions pour filles délinquantes ou en danger. Les législateurs manifestent, pour la première fois, le souci de prendre spécifiquement en charge une population négligée jusque-là ${ }^{4}$.

Ce sont les étapes de cette prise en charge que nous décrirons dans cet article ${ }^{5}$. En premier lieu, nous présenterons les étapes qui ont progressivement conduit à la construction du problème de l'enfance et à l'établissement, à partir de 1869 , d'institutions spécialisées visant à mieux contrôler certains groupes d'enfants. Nous évoquerons ensuite le cas de l'une d'elles, l'Institut Saint-Antoine de Montréal, dont l'histoire illustre l'évolution de la notion même d'enfance à la fin du $\mathrm{XIX}^{\mathrm{e}}$ siècle.

\section{Mise en place des premières politiques pénales envers les mineurs au Québec}

Dès le début du XIX ${ }^{\mathrm{e}}$ siècle, la plupart des pays occidentaux subissent de profondes transformations dans le domaine économique et social, transformations qui vont notamment se manifester par une industrialisation et une urbanisation massives. Dans ce contexte de changements, certains groupes d'enfants vont paraître plus menacés. Au Québec, c'est la présence dans les villes d'enfants abandonnés et orphelins de plus en plus nombreux qui va d'abord attirer l'attention, car ils commencent à poser problème.

\section{La «découverte 》de l'enfance malheureuse et de l'enfance en prison}

À Montréal, l'arrivée massive d'immigrants britanniques au début des années 1820 va entraîner une première spécification de l'enfance (Fecteau et al., 1998: 78). Cette vague amène dans la ville un grand nombre d'orphelins dont le sort inquiète les groupes philanthropiques. La création des premiers orphelinats gérés par des groupes privés témoigne d'une prise de conscience plus aiguë de la situation des enfants malheureux et abandonnés. Toutefois, c'est le sort des enfants coupables et délinquants qui va susciter le plus d'intérêt et mettre en branle tout un ensemble de réformes préludant à l'établissement de politiques pénales spécifiques. À Montréal, au début du XIX ${ }^{\mathrm{e}}$ siècle, la plupart des jeunes délinquants sont enfermés dans la maison d'industrie de Montréal (fondée en 1818) ou dans la prison commune, où jeunes et adultes subissent le même régime. Cette promiscuité va progressivement être jugée néfaste par certains philanthropes, qui réclameront la séparation des enfants et des adultes et la mise en place d'institutions destinées aux plus jeunes. Ce mouvement en faveur de réformes pénitentiaires touche alors un grand nombre de pays occidentaux comme la France, l'Angleterre, les Pays-Bas, la Belgique et les ÉtatsUnis. Dès les années 1830 , de nombreux philanthropes y dénoncent la cohabitation des enfants et des adultes dans les prisons et préconisent des réformes visant à séparer les détenus suivant les catégories d'âge (Dupont-Bouchat et al., 1995 : 10).

\section{Débats et controverses autour des premiers projets d'institution pour jeunes délinquants}

Au Canada, le projet de créer une institution pour jeunes délinquants est abordé en 1843 à l'Assemblée législative. Les membres de l'Assemblée se penchent sur la possibilité d'établir une ou plusieurs maisons de refuge pour 
les délinquants juvéniles afin d'enrayer la progression du crime $^{6}$. $\mathrm{Au}$ terme des débats relatifs à cette question, l'Assemblée législative reconnaît les effets néfastes de la cohabitation entre enfants et adultes dans les prisons et nomme sept membres chargés d'évaluer la possibilité de fonder un ou plusieurs asiles pour jeunes délinquants. Ce projet tournera court : le comité nommé par l'Assemblée sera rapidement dissous et il faudra attendre dix ans pour que se manifeste à nouveau une volonté politique de prise en charge des jeunes délinquants (Fecteau et al., 1998 : 80 ). En 1850 , le député bascanadien Price soumet un projet de loi pour améliorer le système de discipline, rendre l'administration des prisons plus économique et permettre la construction et le maintien d'une maison de correction pour délinquants juvéniles. Le projet, abandonné une première fois, est présenté de nouveau et corrigé l'année suivante. Cette fois, le député Price propose la création de deux institutions destinées aux jeunes délinquants, l'une pour le Haut-Canada (territoire actuel de l'Ontario), l'autre pour le BasCanada (le Québec). Ce projet est également abandonné?

Malgré tout, la volonté d'implanter un régime spécial de traitement des jeunes délinquants continue de se manifester. En 1852, le gouverneur Elgin demande à deux inspecteurs du pénitencier provincial $^{8}$, Wolfred Nelson et Andrew Dickson, d'enquêter sur l'état des prisons de la colonie. Au terme de leurs investigations, les deux inspecteurs soulignent encore une fois l'urgente nécessité d'une réforme des prisons. Ils proposent aussi la création de programmes spéciaux pour les jeunes délinquants et les enfants abandonnés. Les conceptions des deux inspecteurs divergent cependant quant aux modalités d'application de ces programmes ${ }^{9}$. Pour Dickson, la solution au problème de l'enfance malheureuse et délinquante passe par la création d'institutions spéciales de réforme. À l'opposé, Nelson prône l'établissement d'un réseau d'apprentissage et la création de fermes modèles en vue de réinsérer socialement les jeunes déviants. Mieux vaut, selon lui, promouvoir l'éducation des masses et favoriser par ce biais la prévention du crime.

L'opposition de Nelson à la mise en place d'institutions aura pour effet immédiat d'empêcher l'adoption de toute loi concernant le traitement de la délinquance juvénile, le conflit entre les deux inspecteurs incitant les autorités à remettre les débats à plus tard. Cependant, le sort des enfants en prison et le problème de la délinquance juvénile continuent de préoccuper une bonne partie de l'opinion. À la fin des années 1850 , certains groupes d'enfants et d'adolescents deviennent plus visibles et surtout plus «menaçants»: jeunes «oisifs» hantant les rues des métropoles, vagabonds et petits voleurs dont la présence perturbe l'harmonie sociale. Leur nombre de plus en plus élevé vient souligner la nécessité d'intervenir et d'accorder à ces populations un traitement distinct. Au début de 1857, une séric de sept lettres signées Philanthropy relance le débat. L'auteur, s'adressant au directeur du journal anglophone Montreal Gazette, reproche aux gouvernants leur lenteur et leurs hésitations en matière de traitement des enfants vagabonds et délinquants. Il insiste notamment pour que les autorités prennent enfin leurs responsabilités et adoptent des lois visant la prévention et le traitement de la délinquance juvénile ${ }^{10}$.

L'Assemblée répond enfin aux sollicitations et sanctionne, le 10 juin 1857 , l'Acte pour établir des prisons pour jeunes délin-

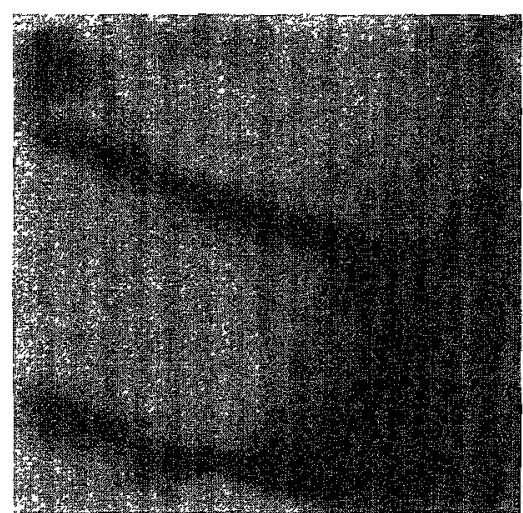

quants, pour la meilleure administration des asiles, hôpitaux et prisons publics, et pour mieux construire des prisons communes ${ }^{11}$. La loi prévoit notamment la création de deux prisons de réforme pour les mineurs âgés de moins de 21 ans, l'une dans le Haut-Canada, l'autre dans le Bas-Canada. Ces deux prisons seront dirigées par un préfet, assisté d'un bureau d'inspecteurs. En prenant ces mesures, les autorités manifestent pour la première fois le désir de faire sortir les jeunes des prisons et des pénitenciers afin de les placer dans des institutions susceptibles de les réformer à long terme.

\section{La prison de réforme de l'île-aux-Noix (1858-1862)}

La prison de réforme du BasCanada fut officiellement ouverte en 1858. Elle occupait les bâtiments d'une ancienne caserne militaire de l'île-aux-Noix, sur la rivière Richelieu. Le préfet désigné pour diriger la prison de réforme était Andrew Dickson, l'ancien inspecteur des pénitenciers. Cette première expérience d'institutionnalisation des mineurs se révélera très rapidement désastreuse. Le bâtiment n'étant pas muni des dispositifs nécessaires à l'enfermement et le personnel étant peu expérimenté, on comptera 19 évasions durant la première année de fonctionnement de la prison ${ }^{12}$. Outre la mauvaise 
organisation des lieux, la population pose aussi problème. La présence d'un grand nombre de jeunes criminels endurcis en son sein et les difficultés liées à la cohabitation des filles et des garçons sont d'importants facteurs de perturbations ${ }^{13}$. Les problèmes de discipline sont aussi liés au conflit opposant le préfet au personnel, qui est en outre peu qualifié pour effectuer les tâches exigées ${ }^{14}$. Après le départ de Dickson, en 1860, la situation ira encore en se détériorant. Le nouveau préfet, François-Xavier Prieur, devra faire face à plusieurs émeutes graves parmi les jeunes détenus et fera l'objet de virulentes attaques à cause, notamment, de son recours trop fréquent aux châtiments corporels. Finalement, l'expérience de l'T̂le-aux-Noix s'étant révélée bien en deçà des attentes initiales, la prison sera fermée et déménagée en 1862 à Saint-Vincent-de-Paul. Ici encore, l'enfermement des jeunes délinquants dans l'établissement ne donnera pas les résultats de réforme escomptés.

La critique des prisons de réforme se fait plus vive au moment où la peur monte parmi les élites des sociétés occidentales face au phénomène de l'errance des jeunes dans les rues des villes. Ces jeunes, surnommés par plusieurs «Arabes des villes », sont généralement des enfants abandonnés ou des petits vagabonds qui n'ont pas commis d'acte criminel suffisamment grave pour être condamnés à purger une peine d'enfermement. La crainte qu'ils suscitent rend caduc et obsolète le ciblage des seuls jeunes délinquants. La nécessité perçue auparavant d'élargir le bassin de jeunes susceptibles d'être traités devient donc de plus en plus urgente.

La plupart des sociétés occidentales adopteront ainsi des mesures visant à résoudre ce problème. Il s'agit alors d'élargir les critères d'intervention en matière d'enfance en danger. Dans un premier temps, l'établissement de critères permettant la distinction entre les jeunes délinquants et les jeunes ayant besoin de protection permettra d'atteindre cet objectif. Dans cette optique, des réformateurs comme Charles L. Brace ${ }^{15}$ rendent possible une véritable «politique» de l'enfance déviante centrée sur deux stratégies souvent rivales: l'enfermement et le placement en foyer nourricier (Fecteau, 1998: 130). Certains pays, comme les ÉtatsUnis, privilégient de plus en plus cette dernière approche pour traiter les cas d'enfants non criminels jugés en danger. D'autres pays, comme l'Angleterre, privilégient l'approche institutionnelle.

\section{La création des écoles de réforme et d'industrie}

Les lois de 1869 établissant les écoles de réforme et d'industrie ${ }^{16}$ au Québec s'inspirent du modèle anglais ${ }^{17}$. L'approche institutionnelle développée en Angleterre convient aux législateurs québécois parce qu'elle permet l'établissement d'un système privé d'écoles de réforme et d'industrie financé par l'État. Un tel système offre plus de flexibilité qu'un système d'enfermement public, en permettant notamment d'adopter certaines orientations relatives à la religion des jeunes détenus (Ménard, 1998b : 19-29). Alors que la loi établissant les écoles d'industrie fixe les critères de prise en charge des enfants jugés en danger, la loi établissant les écoles de réforme se veut une réponse aux critiques qui étaient formulées contre l'enfermement des jeunes délinquants à la prison de réforme. Ainsi, les législateurs québécois tentent de remédier au caractère pénal de ces institutions en permettant que des écoles de réforme reconnues soient établies sur leur territoire ${ }^{18}$. Ces dernières devaient offrir des conditions d'enfermement plus personnalisées que les premières, leurs directeurs pouvant faire leurs propres règlements, pourvu que ceux-ci soient approuvés par le Lieutenantgouverneur ${ }^{19}$.

Le Québec suit ainsi la tendance occidentale visant à uniformiser le traitement des jeunes délinquants et des jeunes en danger (Dupont-Bouchat et al., 1996; Digneffe et Dupont-Bouchat, 1982: 31-165). Ces deux catégories d'enfants sont envoyés dans des lieux différents, mais on considère dorénavant qu'ils requièrent le même type d'intervention. L'histoire de la prise en charge de l'enfance sera, dès lors, de plus en plus axée sur l'extension du pouvoir de l'État et de ses mandataires à l'endroit des mineurs et, éventuellement, de leur famille. La mise sur pied d'institutions d'enfermement qui leur sont destinées va servir à atteindre ce but. En général, elle coïncide, comme l'illustre bien le cas du Québec, avec l'adoption de lois qui étendent le pouvoir de contrainte de l'État sur les mineurs. En fait, plus l'enfermement des jeunes dans des institutions spécialisées se répand, plus l'adoption de mesures permettant d'exercer sur eux une contrainte devient la norme ${ }^{20}$. Cependant on assiste, parallèlement à ce phénomène, à la montée d'autres mouvements qui mettent en question la place centrale de 


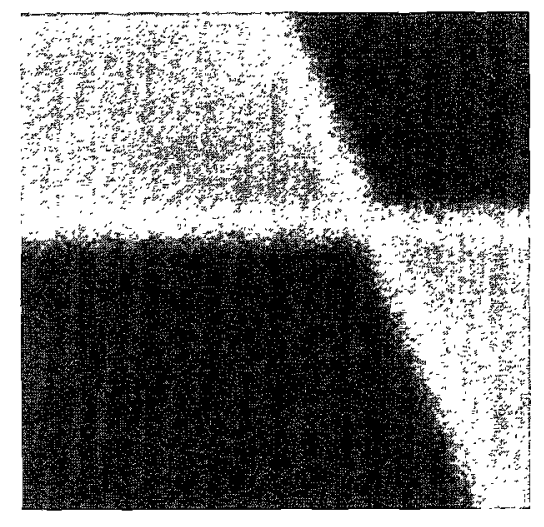

l'institutionnalisation dans la réforme des jeunes.

\section{L'exemple de l'Institut}

Saint-Antoine (1873-1912)

L'exemple de l'Institut SaintAntoine pour garçons délinquants, qui demeure durant toute la période la plus importante institution d'enfermement pour les mineurs de la province, sera retenu ici pour illustrer comment le Québec vit cette évolution ${ }^{21}$. L'expérience de l'institutionnalisation spécialisée dans la réforme des jeunes va effectivement jouer un rôle important dans la lutte menée pour élargir le pouvoir de contrainte de l'État sur cette clientèle ${ }^{22}$. En ce sens, l'expérience québécoise est marquée par les débats occidentaux qui remettent en question l'efficacité de l'institutionnalisation comme moyen de réformer les jeunes ${ }^{23}$.

\section{Un régime de vie plus «paternel»}

C'est ainsi que lors de son ouverture, en 1873, l'Institut SaintAntoine présente plusieurs des caractéristiques propres aux nouvelles institutions pour mineurs qui se sont implantées ailleurs en Occident. Celles-ci sont censées être plus humaines que les anciennes prisons pour enfants, leurs méthodes éducatives devant ressembler le plus possible à celles qui sont appliquées dans les familles.
La transition de la prison vers l'école est notamment perceptible dans certains changements ayant trait à l'aspect extérieur de l'Institut Saint-Antoine. En effet, l'école de réforme présente au public une image moins rigide que l'ancienne prison de réforme en raison de l'absence de grillages aux fenêtres, de portes de fer et de murs d'enceinte imposants. De plus, un régime en commun remplace l'ancien régime cellulaire, les jeunes passant désormais la nuit dans des dortoirs au lieu d'être enfermés pour dormir ${ }^{24}$. Les frères se targuent également d'appliquer un régime paternel envers les jeunes détenus. Les punitions corporelles sont officiellement bannies de l'établissement, tandis que l'enfermement solitaire, utilisé comme moyen de punition, est censé favoriser la moralisation ${ }^{25}$.

Cette volonté de moraliser révèle que la bienveillance des frères vise à former de futurs citoyens dociles et productifs. En fait, le modèle paternel implique qu'une discipline sévère encadre les enfants. Leur vie quotidienne est réglée jusque dans les moindres détails et ils sont l'objet d'une surveillance constante. De plus, c'est par le travail que l'on veut parvenir à réformer les jeunes détenus. Le temps consacré à leur formation scolaire demeure minime durant toute la période et les jeunes passent la majeure partie de leur temps à travailler dans des manufactures où on est censé leur apprendre un métier. L'objectif de la formation, clairement énoncé par le directeur de l'établissement, est de donner au pays un grand nombre de bons ouvriers ${ }^{26}$. Dans les faits, la formation offerte dans les manufactures est limitée et les jeunes détenus servent de main-d'œuvre à bon marché aux entrepreneurs qui louent des locaux aux frères.

C'est pour ces raisons que l'Institut Saint-Antoine devient l'objet de critiques de plus en plus vives, à l'instar des institutions «familiales» établies ailleurs en Occident. Des réformateurs soulignent ainsi que ces institutions semblent continuer de fonctionner comme des prisons ou des pénitenciers pour jeunes et qu'on s'éloigne dans la pratique du discours ayant mené à leur création. Il en résulterait un grand nombre de récidives (Dupont-Bouchat et al., 1996: 290-312; Schlossman, 1977: 105-123).

\section{Remise en question du modèle institutionnel}

Avec la montée de ces critiques, les discours contestant l'efficacité de l'enfermement comme moyen de prévenir les déviances, qui se sont développés depuis les années 1850 , pourront prendre de plus en plus de place. Vers les années 1880 , à l'occasion des multiples congrès internationaux qui se déroulent en Europe et aux États-Unis, des réformateurs intensifient leur lutte contre l'institutionnalisation ${ }^{27}$. Les discours seront dès lors de plus en plus axés sur la nécessité de prévenir et de protéger en s'attaquant le plus directement à la source du problème de la délinquance juvérile, soit les parents inaptes (Trépanier et al., 1995: 32-33). C'est ainsi que des réformateurs proposent l'adoption de mesures qui pourraient permettre de garder le plus possible les jeunes déviants dans leur famille. En plus d'assurer un meilleur contrôle des familles, ces mesures permettraient de mieux tenir compte de la diversité des cas et des histoires familiales. De plus en plus de sociétés de patronage, ayant pour but de trouver un substitut familial réel aux jeunes abandonnés et même aux jeunes délinquants, sont également mises sur pied en Europe; elles ont des équivalents ailleurs, telles les sociétés d'aide à l'enfance en Ontario ${ }^{28}$. 
Les autorités responsables des institutions d'enfermement pour les jeunes réagiront aux critiques contre leur inefficacité en adaptant leurs pratiques aux nouveaux discours sur la réforme. Elles tentent alors de démontrer que la spécialisation des traitements offerts aux mineurs peut s'effectuer à l'intérieur des murs de leur établissement. À l'Institut Saint-Antoine, on suit ainsi la tendance à personnaliser de plus en plus les traitements destinés aux jeunes détenus. Ces derniers deviennent l'objet d'une classification de plus en plus fine et on implante un système de bons points apparenté à celui des sentences à durée indéterminée qui entre en vigueur dans la plupart des institutions d'enfermement ${ }^{29}$. En faisant dépendre de leur comportement la durée de détention des jeunes détenus, on espère obtenir de meilleurs résultats de réforme. Les récompenses qui s'ajoutent aux punitions n'ont donc pas pour but d'humaniser les traitements.

Ce système n'est toutefois officialisé par une loi qu'en $1903^{30}$, alors que les frères le réclament depuis l'ouverture de leur institution, tout en reprochant au gouvernement de miner leur autorité auprès des jeunes détenus en accordant trop de libérations sur requête des parents ${ }^{31}$. Les frères réclament également la mise sur pied de sociétés de patronage dès les années 1880 , conformément au dis- cours sur la récidive qui se tient ailleurs en Occident. De telles sociétés ne verront toutefois jamais le jour au Québec, malgré les demandes répétées de la direction de l'Institut Saint-Antoine. Les législateurs québécois ne suivent ainsi que très lentement la tendance à élargir le pouvoir de contrainte de l'État sur les familles. Si les lois établissant les écoles de réforme et d'industrie s'inscrivent dans la logique de l'investissement graduel de l'État dans le lien parental, les législateurs québécois enferment cet investissement dans des balises étroites. Le pouvoir de contrainte s'exercera, durant toute cette période, dans les strictes limites institutionnelles de la prison de réforme, puis des écoles de réforme et d'industrie, sous la surveillance des inspecteurs des prisons et asiles $^{32}$.

\section{L'implantation des tribunaux pour mineurs}

Malgré son choix institutionnel, le Québec finira par suivre la tendance occidentale observée au tournant des $\mathrm{XIX}^{\mathrm{c}}$ et $\mathrm{XX}^{\mathrm{e}}$ siècles, en adoptant en 1910 une loi permettant la mise en place de tribunaux pour mineurs ${ }^{33}$. Cependant, en dépit des critiques émises contre l'école de réforme des garçons de Montréal, l'établissement des tribunaux pour mineurs au Québec ne découle aucunement, comme en Ontario, d'une remise en question du rôle de l'institutionnalisation comme moyen de réformer les déviances des jeunes. Dans la province voisine, le journaliste $\mathbf{J}$. J. Kelso devient en 1893 le premier surintendant des enfants négligés, après avoir été à la tête d'un mouvement farouchement opposé au placement institutionnel et prônant plutôt le placement familial ${ }^{34}$. L'idée de recourir à des approches non institutionnelles pour les enfants en danger et même pour les mineurs délinquants, qui gagne du terrain en Ontario et ailleurs, mène à l'institution de la probation, qui sera vue comme un élément important de la justice des mineurs. En effet, la loi fédérale de 1908 sur les jeunes délinquants ${ }^{35}$ permet aux provinces qui le désirent d'établir un tribunal pour mineurs ${ }^{36}$, allant dans le sens de l'approche protectrice adoptée par l'Ontario, qui ne pouvait étendre cette approche aux auteurs d'infractions aux lois fédérales, c'est-à-dire à la quasi-totalité des mineurs délinquants (Trépanier et al., 1995: 23; Dubois et al., 1999: 345). Cette loi institue un régime de probation dont les agents apparaissent, pour les jeunes délinquants, comme le pendant des agents d'aide à l'enfance chargés, en Ontario, d'intervenir auprès des enfants en danger. Quant aux juges pour enfants, ils vont permettre d'étendre la même approche protectrice aux enfants délinquants (Dubois et al., 1999: 346).

L'agent de probation devient ainsi une composante essentielle du nouveau système (Trépanier et al., 1995: 46). Sa fonction est double. Dans un premier temps, il rend possible l'application de mesures qui tiennent compte des besoins de l'enfant et des résultats de réforme, en faisant enquête sur la famille et en formulant ses recommandations au juge qui doit décider de la mesure probatoire. Dans le cas d'un premier délit, ce dernier choisit souvent de suspendre cette mesure jusqu'à ce qu'il y ait récidive. Si un second délit est commis, le juge peut décider d'appliquer une mesure probatoire active et fait de nouveau appel à l'agent. $\mathrm{Ce}$ dernier peut alors s'introduire dans le domicile et voir à ce que les parents offrent à l'enfant le milieu de vie jugé adéquat, quitte à brandir la menace d'un placement si nécessaire (Trépanier et al., 1995: 34). Cette fonction de surveillance permet un meilleur contrôle des familles 
estimées inaptes. Ainsi, les tribunaux pour mineurs n'entraînent pas la disparition de l'institutionnalisation. Au contraire, leur mise sur pied ne fait que concrétiser un peu plus la nécessité de traiter les jeunes déviants selon leurs besoins. L'institutionnalisation peut parfois être la meilleure réponse aux besoins d'un jeune alors que, pour un autre, le placement en famille d'accueil est préférable. En ce sens, la mise en place des tribunaux pour mineurs contribue à légitimer le placement en institution et toute autre mesure visant à guérir les symptômes de la délinquance (Rothman, 1980: 259). L'objectif officiel de l'ouverture de la Cour des jeunes délinquants de Montréal en 1912 était ainsi de favoriser le recours à la probation plutôt que le placement en institution. En pratique, cette Cour a permis que les deux solutions se complètent: les mesures probatoires sont introduites, mais la clientèle des écoles de réforme croît également, si bien que les institutions voient augmenter leurs budgets et s'améliorer leurs installations. Durant les huit années suivant l'ouverture de la Cour des jeunes délinquants de Montréal, le nombre d'enfants inscrits dans les écoles de réforme du Québec croît, passant de 367 en 1911 à 678 en 1919 (cette augmentation survient surtout lors de la Première Guerre mondiale) (Rains et al., 1992: 21-22) ${ }^{37}$. La situation est similaire en Ontario: en dépit de l'apparente victoire de Kelso contre l'institutionnalisation, la majorité des représentants gouvernementaux, des magistrats et des juges continuent d'y voir un moyen efficace pour réformer les garçons délinquants (Jones, 1978 : 242).

L'établissement de ces tribunaux a donc notamment pour but de faciliter la mise en place de mesures en milieu ouvert pour les jeunes déviants. Ces mesures vont de pair avec la probation, laquelle est généralement considérée comme un accessoire essentiel des tribunaux pour mineurs. Si les critiques contre l'enfermement ont eu pour effet d'entraîner ces changements d'orientation dans le système de justice des mineurs, la mise en place de tribunaux spécifiquement destinés aux jeunes constitue également l'aboutissement de la démarche visant à élargir le pouvoir de contrainte de l'État sur ces derniers. Dans cette optique, on peut affirmer que les critiques émises contre l'enfermement donnent à l'État l'occasion de créer des mesures qui lui permettent d'intervenir directement auprès des familles. Ces tribunaux permettent en effet d'amenuiser ou d'abolir selon les endroits où ils sont établis $^{38}$ - les distinctions qui subsistaient entre les jeunes criminels et les jeunes en danger, en instituant un modèle de protection applicable aux deux catégories. La mise en place des tribunaux pour mineurs consacre donc la rupture avec le droit pénal classique puisqu'elle remplace, pour les mineurs, une approche fondée sur la punition par une autre où la protection de l'enfance apparaît comme le meilleur moyen de protéger la société (Trépanier et al., $1995: 44)$.

La création des tribunaux pour mineurs prive ainsi les mineurs délinquants ou en danger des garanties que la procédure pénale accorde aux délinquants adultes. $\mathrm{La}$ fonction même du tribunal, où on est censé travailler dans l'intérêt du mineur (Trépanier et al., $1995:$ 47), entraîne cet état de fait. Toutes les formes d'enfance déviante peuvent désormais devenir objet de régulation par les instances étatiques.

\section{Conclusion}

En Europe et en Amérique du Nord, le XIX ${ }^{\mathrm{e}}$ siècle est une période charnière dans le développement des idées et la transforma-

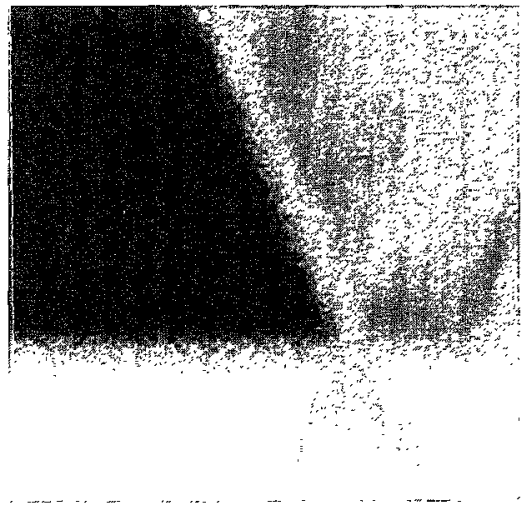

tion du regard sur l'enfance malheureuse. L'étude menée dans le cadre de cet article a permis de saisir les étapes de ces changements, qui font apparaître les premières politiques spécifiques à l'enfance au XIX ${ }^{\mathrm{e}}$ siècle et au début cu XX $\mathrm{XX}^{\mathrm{e}}$ siècle. Durant cette période, l'enfant sera progressivement perçu comme un sujet à part, ayant des besoins qui le distinguent des adultes. Au Québec, cette évolution se découpe en trois grandes périodes (Fecteau, 1998 : 130-131).

Durant les années 1820-1840, c'est tout d'abord l'enfance urbaine, abandonnée ou orpheline, qui interpelle et dérange. À Montréal, la création d'orphelinats gérés par des groupes privés témoigne de cette première volonté

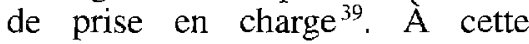
époque, I"enfant ne se distingue de l'adulte que dans la mesure où son jeune âge exige un traitement différent. En ce sens, les premières mesures prises à l'égard de l'enfance à problèmes visent avant tout à éviter la promiscuité entre enfants et adultes. Ce souci préside à la création des premiers orphelinats de Montréal mais aussi des premiers quartiers de jeunes dans les prisons de France et de Belgique et des premières maisons de refuge de New York, Boston et Philadelphie $^{40}$.

La seconde période, qui débute dans les années 1840 et s'étend jusqu'à la fin du XIX ${ }^{e}$ siècle, voit 
apparaître de nouvelles préoccupations à l'égard de l'enfance. Sous l'influence des théories pédagogiques de Pestalozzi et de Fellenberg, on reconnaît que les enfants se distinguent des adultes en raison de leur âge mais aussi et surtout en raison de leur caractère réformable (Fecteau, 1998: 130). En effet, contrairement aux adultes déviants, sur lesquels les programmes d'intervention n'ont que peu d'impact, les enfants semblent malléables et transformables. On peut donc nourrir plus d'espérances à leur égard. De plus, dans la mesure où ces enfants sont les citoyens de demain, il faut accorder une grande attention à leur éducation et à leur formation (Ménard, 1998b: 19; Strimelle, 1998 : 64). Au Canada, la question des enfants délinquants retenus en prison avec les adultes sera à l'origine des première ébauches d'une politique spécifique à l'enfance. En effet, la volonté de mettre au point un régime spécial de traitement des jeunes délinquants naît de la prise de conscience que ces jeunes, à cause de leur âge et de leur caractère malléable, doivent être pris en charge dans des institutions spéciales afin d'être à l'abri des mauvaises influences des adultes. Dans le cadre des prisons, cette lente prise de conscience conduira au Canada à la création des premières institutions spécialisées pour mineurs délinquants, en 1857. Par la suite, la création d'établissements distincts pour les enfants délinquants et abandonnés favorisera la prise en charge de nouveaux groupes d'enfants qui échappaient jusqu'alors au contrôle des autorités. Dans ce contexte où l'enfant est désormais perçu comme une «valeur» à protéger et à rentabiliser, le rôle de la famille et particulièrement des parents fait aussi l'objet d'une attention accrue. À la fin du XIX ${ }^{e}$ siècle, le besoin de protéger les enfants encouragera la mise en place de politiques favorisant l'intervention auprès des familles dont les compétences éducatives sont mises en doute. Dans cette perspective, l'enfance apparaît de plus en plus comme un enjeu social et politique majeur permettant à l'État de mieux contrôler et surveiller les familles des milieux populaires, jusqualors inatteignables $^{41}$.

La troisième période, qui débute en 1908 avec la loi fédérale sur les jeunes délinquants, voit l'aboutissement de tout le processus de prise en charge de l'enfance et de la famille enclenché durant la période précédente. Cette nouvelle loi, qui prévoit notamment la création de tribunaux pour mineurs, entend développer une approche plus protectrice et plus englobante de la jeunesse en difficulté. Désormais, tous les enfants, qu'ils soient délinquants, abandonnés ou «en danger», peuvent faire l'objet de mesures de prise en charge. Dans ce contexte où la volonté de protéger l'enfant et la méfiance envers les milieux populaires prennent le pas sur le désir de maintenir l'institution familiale intacte, la thématique de l'enfant en danger justifie la surveillance des familles jugées «suspectes» et l'intervention directe auprès d'elles (Quincy-Lefebvre, 1997: 270; Fecteau et al., 1998: 109; Ménard, 1998a: 282). Parallèlement, au tournant du siècle, le développe- ment des sciences médico-psychologiques modifie profondément le statut de l'enfance en la faisant sortir définitivement du monde des adultes et en faisant d'elle un objet particulier de recherches et d'investigation (Gubin, $1984: 86$ ).

Ces modifications profondes du concept même d'enfance auront un double impact sur l'enfant et sur la famille. Devenu l'objet de nouvelles préoccupations et de nouveaux savoirs, le monde de l'enfance subit en effet de nouvelles formes de régulation, qui se développent sous le couvert de mesures protectrices. Cette nouvelle conception de l'enfant remet aussi en cause le rôle de la famille, dont les compétences éducatives peuvent faire l'objet de critiques conduisant éventuellement à des sanctions. Les étapes qui jalonnent la construction de la notion d'enfance doivent donc être lues comme autant de stratégies mises au point par les autorités en vue d'instrumentaliser progressivement les enfants «à problèmes» et leur famille et de faciliter par ce biais la prise en charge de franges de la population jugées incontrôlables et dangereuses. En ce sens, au début du $X X^{\mathrm{e}}$ siècle, le développement de politiques propres à l'enfance relève plus d'un objectif de régulation sociale que d'une volonté bienveillante.

\section{Sylvie Ménard \\ Véronique Strimelle \\ Département de criminologie Université d'Ottawa}

\section{Notes}

Les recherches entreprises au cours des
années 1970 par les historiens et criminolo-
gues de tendance radicale comme Platt
(1977), Ignatieff (1978) ou Cohen et Scull
(1983) ont remis en question l'ancien pos-
tulat suivant lequel les diverses institutions
d'enfermement étaient l'expression d'une
volonté d'humanisation des peines. 
D'après ces études plus critiques, les lois et institutions pénales qui apparaissent au cours du $\mathrm{XIX}^{\mathrm{e}}$ siècle représentaient au contraire des instruments mis en place par le pouvoir afin d'étendre et d'approfondir son emprise sur l'ensemble du corps social. Pour la France, certaines études se sont plutôt attachées aux discours et politiques mis au point par les détenteurs du pouvoir afin de mieux discipliner et normaliser les masses. La prison serait alors une nouvelle technologie du pouvoir visant à domestiquer les corps et surtout à réformer les âmes : Foucault (1975), Perrot (1980)

2 Durant cette période renaît en Europe le mouvement philanthropique, qui s'était déjà développé durant le dernier tiers du siècle des Lumières. Les philanthropes et réformateurs pénitentiaires étaient des laïcs ou des religieux, travaillant dans le domaine privé ou comme fonctionnaires de l'État. Tous ont joué un rôle capital dans la transformation des politiques de correction, tant à l'égard des adultes qu'à l'égard des enfants. Ces réformateurs furent particulièrement nombreux en France ; citons notamment : Charles Lucas, Moreau-Christophe et Demetz. Pour la Belgique, l'inspecteur des prisons Édouard Ducpétiaux fut à l'origine des premières mesures visant à un traitement différentiel des enfants dans les établissements d'enfermement. Pour le Canada, ce sont surtout les inspecteurs des pénitenciers Wolfred NeIson et Andrew Dickson qui favorisèrent la mise en place d'institutions spéciales pour mineurs délinquants : Dupont-Bouchat et al. (1995: 16-50). Les parties de cette recherche concernant le Québec ont été rédigées en collaboration avec les auteures de ce texte, à partir de leur thèse de doctorat en cours. Voir aussi, pour la France, Gaillac (1991)

3 Sur la prison de réforme, voir Ménard (1998a : 60-75) et Strimelle (1998: 76-81).

4 Voir à ce sujet Strimelle (1998 : 91-95).

5 Nous avons pu rédiger cet article grâce à des subventions postdoctorales provenant respectivement du Conseil de recherches en sciences humaines du Canada et du Fonds pour les chercheurs er l'aide à la recherche (Québec). L'article s'inspire en partie de nos thèses de doctorat (Ménard. 1998a ; Strimelle, 1998), qui exploitaient pour la première fois les banques de données constituées par le Centre d'histoire des régulations sociales sur l'émergence et l'évolution historique de I'enfermement à Montréal entre les années 1836 et 1913. Nous souhaitons remercier Jean-Marie Fecteau, professeur au Département d'histoire de l'Université du Québec à Montréal, Jean Trépanier, professeur au Département de criminologie de l'Université de Montréal et chercheur au Centre intemational de criminologie comparée, ainsi que David Niget, étudiant au doctorat raltaché à l'Université d'Angers et à l'Université du
Québec à Montréal, pour leurs précieux conseils.

- Débats de l'Assemblée législative du Canada-Uni, 1843, p. 381.

7 Journaux de l'Assemblée législative du Canada-Uni, 1850, vol. 9, p. 156, et 1851, vol. 10, p. 23. Rappelons ici qu'aux termes de l'Acte d'union de 1840 , les députés du Canada-Est (Bas-Canada) et du CanadaOuest (Haut-Canada) siégeaient à l'Assemblée législative du Canada-Uni

${ }^{8}$ Le pénitencier provincial de Kingston avait été créé en 1835 et accueillait mineurs et adultes condamnés à une peine de plus de deux ans depuis 1841. Dans cet établissement, la seule classification opérée alors était celle distinguant les femmes des hommes.

9 Rapports d'Andrew Dickson et de Wolfred Nelson sur les prisons du Canada-Ouest pour 1852, Journaux de l'Assemblée législative du Canada-Uni (1852-1853), vol. 11, no 4, append. HH.

${ }^{10}$ Philanthropy, Care of our Destitute and Criminal Population, Montréal, Salter \& Ross, 1857 (ICMH 47078), p. 7.

11 S. C. 20 Vict. c. 28.

12 Rapport préliminaire des inspecteurs de prison pour 1859, Journaux de l'Assemblée législative du Canada-Uni (1860), vol. 18, D. S. 32, p. 23

${ }^{13}$ Quelques jeunes filles avaient été admises à l'institution de l'île-aux-Noix lors de son ouverture, parce que la loi établissant les prisons de réforme ne prévoyait pas d'institutions propres à chaque sexe. Celles-ci ne seront toutefois plus admises à la prison de réforme dès 1860, leur présence étant jugée dérangeante pour les autres prisonniers. Les filles condamnées à purger une peine d'enfermement seront internées dans des institutions pénales pour adultes jusqu'à l'adoption de la loi établissant les écoles de réforme, en 1869.

14 Ibid., p. 33.

15 En 1853, Charles Loring Brace fonde la New York Children's Aid Society, qui s'occupe de placer les enfants en famille d'accueil. Inspiré des théories du pédagogue suisse Johann Pestalozzi, Brace croit que les enfants ont des dispositions pour faite le bien et il ne voit pas pourquoi il faudrait les incarcérer. Il favorise également le placement des jeunes à la campagne afin de les éloigner des tentations de la ville.

${ }^{16}$ Loi concernant les écoles de réforme, $\mathrm{S} . \mathrm{Q}$. 32 Vict., c. 18, et Loi concernant les écoles d'industrie, S. Q. 32 Vict., c. 17. II faut souligner ici qu'à la suite de la Confédération canadienne (1867), les gouvemements provinciaux deviennent responsables des institutions pénales, à l'exception des pénitenciers.
17 Pour une analyse du modèle anglais en matière d'enfermement, voir Stack (1979) et Hurt (1984)

${ }_{18}$ Loi concernant les écoles de réforme, S. Q. 32 Vict., c. 18, article 2 .

19 Ibid.

20 J. R. Sutton (1983 : 915-947) a démontré ce phénomène pour les États-Unis.

${ }^{21}$ Au Québec, c'est le problème posé par la délinquance des garçons qui déterminera les politiques adoptées par le gouvernement. C'est la raison pour laquelle nous avons privilégié l'exemple de l'Institut Saint-Antoine pour garçons délinq̨uants. Voir Ménard (1998a : 12)

${ }^{22}$ Françoise Digneffe et Marie-Sylvic Dupont-Bouchat (1982:131-165) ont observé que le nom et l'utilisation des institutions destinées à la réforme des jeunes délinquants en Belgique se modifient en fonction du souci d'adapter les politiques ef les pratiques institutionnelles aux besoins sociaux.

23 Voir Ménard (1998a : 212-275).

${ }^{24} 6^{\mathrm{c}}$ rapporl des inspecteurs des prisons et asiles pour 1873, Journaux de l'Assemblét législative de la Province de Québec (1874), vol. 7 , no 1 , DS 5, p. $72-73$, et $28^{\mathrm{e}}$ rapport des inspecteurs des prisons et asiles pour 1897, Journaux de l'Assemblée législative de la Province de Québec (1898), vol. 32, no 2, DS 9, p. 141.

${ }^{25} \mathrm{G}^{\mathrm{e}}$ tapport des inspecteurs des prisons et asiles pour 1873, op. cit., p. 91.

${ }^{26}$ Rapport du directeur de l'Institut SaintAntoine, le frère Eusèbe, dans le $7^{\mathrm{t}}$ rapport des inspecteurs des prisons et asiles pour 1874 et 1875, Journaux de l'Assemblée législative de la province de Québec (1875), vol. 9, no 1, DS 15, p. 54.

27 Les philanthropes de la fin du siècle cumulent plusieurs fonctions : hommes politiques, juristes, médecins et hommes d'œuvres, ils animent les congrès et les débats parlementaires et président de nombreuses sociétés de bienfaisance. En Belgique, les principaux ténors de la protection de l'enfance sont les miristres de la Justice Jules Lejeune et Henri de Wiart, ainsi qu'Adolphe Prins, inspecteur des prisons du royaume. En France, il y a notamment Théophile Roussel, sénateur et membre de l'Académie de la médecine, Ferdinand Dreyfuss, avocat à la cour de Paris, et Madame Pauline Kergomard, du Conseil de l'instruction publique. Si quelques réformateurs américains assistent régulièrement aux congrès européens (comme H. E Allison et Clarck Bel], respectivement président et secrétaire de la société de médecine légale de New York), iis sont essentiellement tournés vers les États-Unis. Le premier Canadien à se présenter dans un de ces congrès à Washington, en 1910, est le président du 
comité des enfants moralement négligés de l'Ontario, J. J. Kelso : voir Dupont-Bouchat et al. (1995: 252-277).

${ }^{28}$ Pour une analyse de ce phénomène sur la scène européenne, voir Dupont-Bouchat et al. (1995: 252-277).

${ }^{29}$ David J. Rothman (1980) a ainsi montré que la plupart des institutions servant à interner les déviants, qu'il s'agisse des asiles, des prisons ou des écoles de réforme pour jeunes délinquants, doivent adopter ces traitements afin de répondre aux critiques qui les concement.

${ }^{30}$ Loi relative au contrat passé entre le gouvernement et les Frères de la Charité de Saint-Vincent-de-Paul de Montréal, article 9, S. Q., 3 Éd. VII. C. 5.

${ }^{31}$ Notre analyse des données tirées des registres d'écrou de l'Institut Saint-Antoine montre toutefois que l'officialisation du système des bons points n'entraîne pas une diminution des libérations accordées sur requête des parents.

32 Sur ce point, voir également Fecteau et al. (1998: 106-107).

${ }^{33}$ Loi relative aux jeunes délinquants, S. Q., 1 Geo. V, c. 26. Le droit criminel et la procédure criminelle relevant du gouvernement fédéral, la mise en place des tribunaux pour mineurs dans les provinces ne devient possible qu'avec l'adoption de la loi fédérale de 1908 sur les jeunes délinquants. Malgré la loi de 1910 permettant l'établissement de tels tribunaux au Québec, il faut attendre 1912 pour que la Cour des jeunes délinquants de Montréal ouvre officiellement ses portes. Voir Trépanier et Tulkens (1995).

${ }^{34}$ Sur Kelso, voir Jones et al. (1981).

${ }^{35}$ Loi concernant les jeunes délinquants, S. C., 1908, c. 40.

${ }^{36}$ Au Canada, le gouvemement fédéral est responsable du droit et de la procédure criminelle - donc des jeunes délinquants qui ont commis des infractions aux lois fédérales -, alors que les provinces sont responsables des affaires reliées à la protection de l'enfance. L'assimilation des affaires de protection aux affaires de délinquance dépend des lois provinciales.

${ }^{37}$ Nos recherches en cours montrent que la population détenue (nous avons exclu les prévenus de notre anayse) admise à l'Institut Saint-Antoine augmente entre l'ouverture de la Cour des jeunes délinquants et l'époque de la Première Guerre mondiale, passant de 138 en 1912 à 203 en 1919. Nos recherches sur l'institution des Sours du Bon Pasteur pour les filles ne sont pas encore suffisamment avancées pour nous permettre de voir si le même phénomène s'y produit, mais nous savons qu'en 1915 , les fillettes de l'école de réforme et d'industrie quittent définitivement l'institution de Montréal en raison du manque de place, pour s'établir dans les deux institutions de Laval, Moulin du Crochet et Sainte-Domitille. La thèse en cours de David Niget portant sur l'enfant et la justice en France et au Québec pourra aussi nous éclairer éventuellement à ce sujet.

${ }^{39} \mathrm{Si}$ les distinctions entre les mineurs «délinquants » et « en danger» sont abolies au Québec à la suite de l'implantation des tribunaux pour mineurs, il ne faut pas tenir pour acquis que ces tribunaux fonctionnèrent tous de la même manière, et ce même dans les autres provinces canadiennes. À l'extérieur du Canada, les distinctions entre ces deux catégories d'enfants demeurent encore bien présentes en France et en Angleterre, malgré l'implantation de tels tribunaux.

39 Les orphelinats en question étaient: The Montreal Protestant Orphan Asylum (1822), l'Asile des orphelins catholiques (1832), l'Hospice Saint-Joseph (1841), The Saint Patrick Orphan Asylum (1846) et l'Orphelinat Saint-Alexis (1853). Dans ces orphelinats, les principaux gestionnaires étaient des particuliers appartenant à des groupes charitables d'obédience catholique ou protestante : Fecteau et al. (1998: 78).

40 Voir notamment à ce sujet Rothman (1971 : 230-236); Dupont-Bouchat et al. (1995: 51-67) et Dupont-Bouchat et al. (1996: 32)

${ }^{41}$ Sur ce point, voir aussi Dupont-Bouchat (1996: 110)

\section{Bibliographie}

CARLIER, C. 1994. La Prison aux champs. Les colonies d'enfants délinquants du Nord de la France au $19^{e}$ siècle. Paris, L'atelier.

COHEN, S., et A. SCULL 1983. Social Control and the State. Oxford, Robertson.

DIGNEFFE, F., et M.-S. DUPONT-BOUCHAT. 1982. « $\grave{A}$ propos de l'origine et des transformations des maisons pour jeunes délinquants en Belgique au XIX' siècle : l'histoire du penitencier de Saint-Hubert (1840-1890) », Déviance et société, 6, 2: 31-165.

DUBOIS, P. et J. TRÉPANIER. 1999. "L'adoption de la loi des jeunes délinquants de 1908: étude comparée des quotidiens montréalais et torontois », Revue d'histoire de l'Amérique française, 52, 3 : 345-381.

DUPONT-BOUCHAT, M.-S., F. ALEXANDRE et V. STRIMELLE. 1996. De la prison à l'école. Les pénitenciers pour enfants en Belgique au XIX ${ }^{e}$ siecle (1840-1914). Louvain-la-Neuve, UGA.

DUPONT-BOUCHAT, M.-S., J.-G. PETIT, E. PIERRE, B. SCHNAPPER, F. TÉTARD, J. DEKKER, J.-M. FECTEAU et J. TRÉPANIER. 1995. Enfants corrigés, enfants protégés. Genèse de la protection de l'enfance en Belgique, en France, aux Pays-Bas et au Québec (1830-1914). Convention de recherche avec le Ministère français de la Justice : Université d'Angers, Université de Groeningen, Université de Montréal et Université du Québec à Montréal.

FECTEAU, J.-M. 1998. « Note sur les enjeux de la prise en charge de l'enfance délinquante et en danger au XIX' siècle ", Lien social et Politiques-RIAC, 40: 129-138.

FECTEAU, J.-M., S. MÉNARD, V. STRIMELLE et J. TRÉPANIER. 1998. «Une politique de l'enfance délinquante et en danger : la mise en place des écoles de réforme et d'industrie au Québec (18401873) », Crime, histoire et sociétés, II, 1 : 75-110.

FOUCAULT, M. 1975. Surveiller et Punir. Naissance de la prison. Paris, Gallimard.

GAILLAC, H. 1991. Les Maisons de correction, 1830-1945. Paris, Cujas, $2^{\mathrm{C}}$ éd.

GUBIN, E. 1984. * La signification socioéconomique de l'enfant et la famille aux $\mathrm{XIX}^{\mathrm{e}}$-XX' $\mathrm{X}^{\mathrm{e}}$ siècles ", dans $L^{\prime}$ Enfant dans l'art belge de 1800 à nos jours. Bruxelles, CGER.

HURT, J. 1984. «Reformatory and industrial schools before 1933 », History of Education, $13: 45-58$.

IGNATIEFF, M. 1978. A Just Measure of Pain. The Penitentiary in the Industrial Revolution, 1750-1850. New York, Columbia University Press.

JONES, A. 1978. «Closing Penetanguishene reformatory : An attempt to deinstitutionalize treatment of juvenile offenders in early twentieth century Ontario», Ontario History, 70: 227-244.

JONES, A., et L. RUTMAN 1981. In the Children's Aid: J.J. Kelso and Child Welfare in Ontario. Toronto, University of Toronto Press. 
MÉNARD, S. 1998a. L'Institut Saint-Antoine et la problématique de réforme des garçons délinquants au Québec (1873-1910). Université du Québec à Montréal, thèse de doctorat en histoire.

MÉNARD, S. 1998b. «Une politique de l'enfance délinquante : la mise en place de l'école de réforme des garçons de Montréal, 1850-1873 m, Bulletin d'histoire politique, 6, 2 : 19-29.

NIGET, D. L'Enfant et la justice en France et au Québec : 1890-1940. Université d'Angers et Université du Québec à Montréal, thèse en cours.

PERROT, M., dir. 1980. L'Impossible Prison. Paris, Seuil.

PETIT, J.-G. 1990. Ces Peines obscures. La prison pénale en France, 1780-1880. Paris, Fayard.

PLATT, A. 1977. The Child Savers. The Invention of Delinquency. Chicago, University of Chicago Press, $2^{c}$ éd.

QUNNCY-LEFEBVRE, P. 1977. Familles, institutions et déviances. Une histoire de l'enfance difficile, 1880-fin des années trente. Paris, Economica.

RAINS, P., et E. TERAM. 1992. Normal Bad Boys. Public Policies, Institutions, and the Politics of Client Recruitment. Montréal, Kingston, London et Buffalo, McGillQueen's University Press.

ROTHMAN, D. J. 1971. The Discovery of the Asylum. Social Order and Disorder in the New Republic. Boston, Little, Brown.

ROTHMAN, D. J. 1980. Conscience and Convenience. The Asylum and its Alternatives in Progressive America. Boston, Little, Brown \& Co.

SCHLOSSMAN, S. L. 1977. Love and the American Delinquent: The Theory and Practice of Progressive Juvenile Justice, 1825-1920. Chicago, University of Chicago Press.

STACK, J. A. 1979. « The juvenile delinquent and England's "revolution in government" : 1825-1875 », Historians, 42: 42-57.

STRIMELLE, V. 1998. La Gestion de la deviance des filles et les institutions du Bon Pasteur à Montréal (1869-1912). Université de Montréal, thèse de doctorat en criminologie.

SUTTON, J. R. 1983. «Social structure, institutions and the legal status of children in the United States ", American Review of Sociology, 88, 5 : 915-947.

TRÉPANIER, J., et F. TULKENS. 1995. Délinquance et protection de la jeunesse. Aux sources des lois belge et canadienne sur l'enfance. Montréal, Ottawa et Bruxelles, Les Presses de 1'Université de Montréal, Les Presses de 1'Université d'Ottawa et De Boeck Université. 